\title{
A comparison of the behaviour of some root systems under restricted aeration
}

\section{K. Wiersum}

Institute for Soil Fertility, Postbus 30003, 9750 RA Haren-Gr., the Netherlands

Accepted: 4 October 1978

Key words: soil aeration, root development, oats, pea, potato, tomato, tulip, mineral uptake

\section{Summary}

The limiting aeration requirements of a number of crop plants were measured, with oxygen diffusion rate values as a parameter. Tomato and pea were found to have the highest requirements, oats the lowest, while tulip and potato are intermediate. This sequence corresponds rather well with field experience.

Tulip was not found to be especially sensitive to a period of anaerobic conditions.

Shallow rooting as a result of poor soil aeration can restrict the uptake of a number of mineral nutrients. In these experiments, $\mathrm{P}$ and $\mathrm{K}$ are more often severely restricted than $\mathrm{Ca}$ or $\mathrm{NO}_{3}$.

\section{Introduction}

Crop failure or poor productivity of the stand, accompanied by more or less severely damaged root systems, is no uncommon feature in the field. In many cases it seems obvious to suspect poor aeration to be the cause, and this can often be confirmed by subsequent research.

Problems encountered in the bulb-growing industry on heavier soils stimulated research into the aeration requirements of this crop (Boekel, 1971; Wiersum, 1971).

As the measurements of soil aeration and oxygen consumption of roots is part of our research programme, it seemed worth-while to be able to compare the requirements of the tulip with those of a few other crops. Especilaly so, as the tulip root system is considered to be quite sentitive by some of the growers.

The parameter measured to describe the aeration status of the soil was the oxygen diffusion rate (ODR) to a reducing, small, conical, gold electrode. The ODR value gives the highest possible flux of oxygen at a certain spot in the soil to an oxygenconsuming surface, more or less under the same conditions as are valid for a root (Lemon \& Erickson, 1955). It integrates the effect of oxygen diffusion in the soil atmosphere and its diffusion through the water film surrounding the root or electrode. 
The aeration requirements of the root systems were characterized by the growthlimiting ODR value. This value is the measured oxygen flux, expressed in $\mu \mathrm{A}$, at the ultimate depth attained by the root system, under conditions where no other restrictions would prevent deeper penetration. The simple ODR measurements we used do not give unequivocal values irrespective of soil type and other secondary factors, because different electrical resistance of the soil was not taken into account. The effective potential at the electrode will thus be variable. It also makes no sense to give actual oxygen flux values, as our $6 \mathrm{~mm}$ long and conical gold electrode differs in surface from the commonly used platinum-wire electrode.

Another point of interest was to obtain some data on the resistance of root systems to a period of insufficient aeration.

\section{Experimental methods}

Variations in aeration at a given depth in the soil can be brought about by different means, for example by altering soil density or water content. In our case we preferred to use regulation of the soil atmosphere in a setup closely related to that introduced by Stolzy \& Letey (1964), in which the oxygen content of the air above the soil is varied. In this manner soil density and depth of water table are the same in each vessel. The variation imposed is a difference in the depth of the same aeration status (same ODR value), as with the higher oxygen content in the atmosphere a thicker layer of soil can be adequately provided and a longer gradient exists. Thus the roots can grow downward to different depths before encountering limiting ODR.

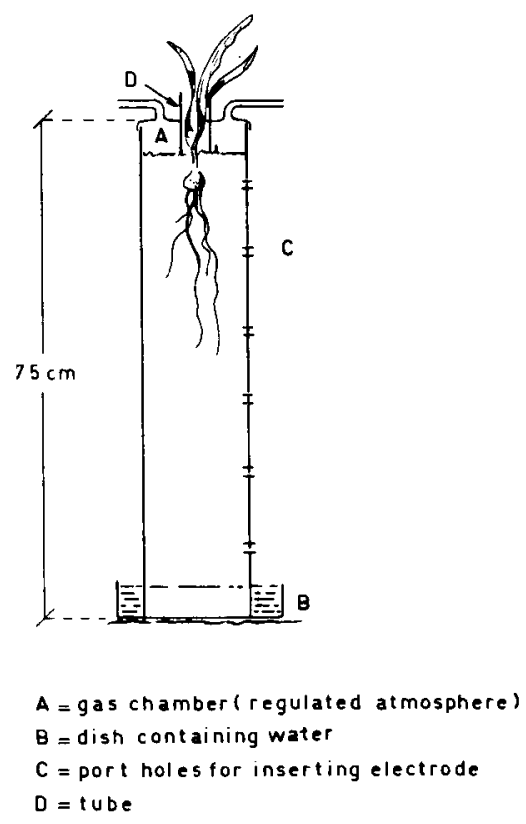

Neth. J. agric. Sci. 27 (1979)
Fig. 1. Diagram of the experimental setup. 


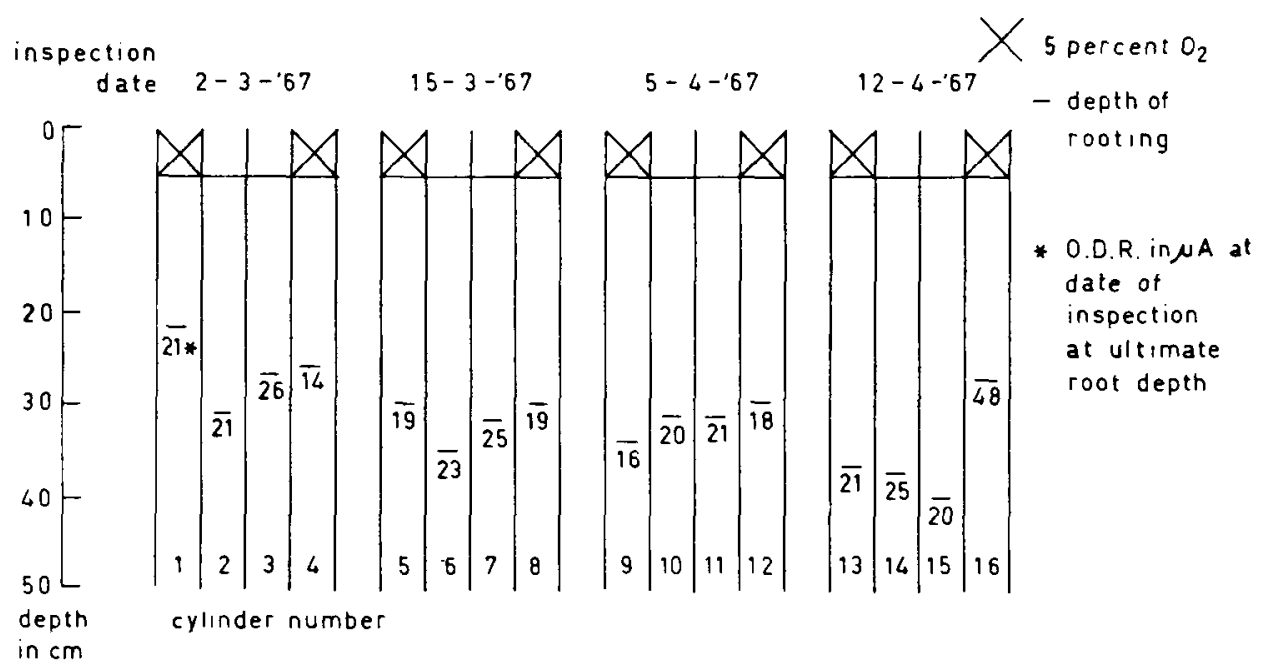

Fig. 2. Root depth attained and the corresponding ODR value in an experiment with tulips.

The experiments were performed in plastic tubes, diameter $15 \mathrm{~cm}$ and generally $75 \mathrm{~cm}$ high. They were placed in dishes continuously filled with water (Fig. 1) and had a number of stoppered holes along the side to allow insertion of the electrodes. The top of the tube is closed by a lid with a narrow pipe extending downward to the soil, allowing the plant to pass through it. The lid had two tubes connected to it, so that the enclosed volume of air above the soil could be flushed with the required air-nitrogen mixture.

This method allows the roots to grow downward undisturbed, until the rate of oxygen supply limits further penetration. Under these standardized conditions clear differences in depth of rooting can be obtained. The limiting ODR can be calculated by interpolation from the values obtained at $10-\mathrm{cm}$ intervals through the holes on the side of the tube. In some cases the variation in the values was within reasonable limits (Fig. 2). But as in the course of a few years different soils were used, densities varied to some extent, and cultural conditions in the glasshouse variesd according to season, many observed values show a large variation. Only seldom were two species compared in the same experiment.

The soils used in these experiments were either a clay loam or a sandy soil. The cylindrical vessels were filled by carefully tamping down the soil to the density as required for each experiment. The ODR measurements were performed with simple equipment, to be described elsewhere, and - except for the conical golden electrode - essentially the same as previously used (Wiersum, 1960). In some cases soil temperature recordings were made by means of a thermograph with the sensor in the center of the soil-filled tube.

At the termination of the treatment the plant was measured and harvested. The plastic pipes were cut open lengthwise so that the root system was clearly exposed. The first measurements of the depth attained were taken (Fig. 3). With a spray gun 


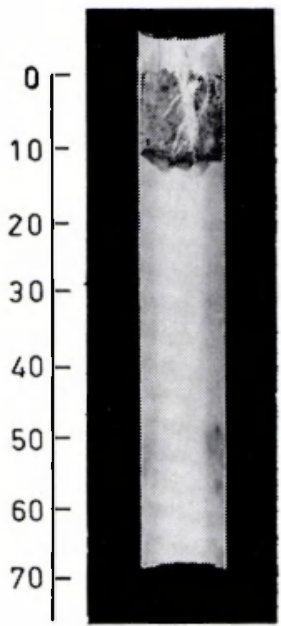

$2.5 \% \mathrm{O}_{2}$

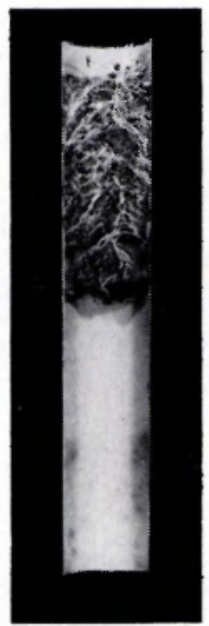

$5 \% \mathrm{O}_{2}$

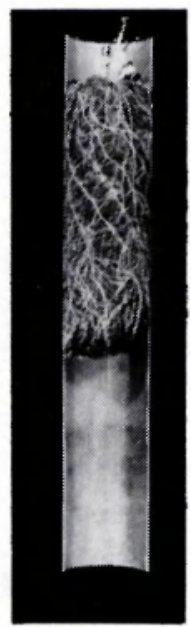

$20 \% \mathrm{O}_{2}$

Fig. 3. Depths attained by potato roots with $2.5 \%, 5 \%$ or $20 \%$ oxygen above the soil surface (clay loam).

the root system was then washed free of soil, measured and then dried for weight determination and analysis.

The different oxygen levels in the air mixture above the soil were obtained by mixing air with the required amount of nitrogen gas. The rates of mixing were regulated by flow meters, as well as the rate of flow through the perfusion chamber at the top of the closed vessel ( 2 litres/hour).

In order to investigate root resistance to lack of oxygen, either a nitrogen atmosphere was applied above the soil or in some cases the water-table was raised above the level that the roots had attained under more favourable conditions before this treatment.

\section{Results and discussion of the experiments}

Two sets of data can be used to evaluate the relative sensitivity of tulip roots to insufficient aeration. The average ODR value obtained at the point of greatest depth attained by the roots can be considered a measure of the lowest acceptable $\mathrm{O}_{2}$ supply under constant conditions. As the experiments were carried out during the course of a few years under different climatic conditions in the glasshouse and

Table 1. Approximate limiting ODR values (in !LA) for root growth of different crops under more or less comparable conditions.

\begin{tabular}{llllll}
\hline Type of soil & Oats & Potato & Tulip & Tomato & Pea \\
Clay loam & 10 & 16 & 17 & - & 20 \\
Sandy soil & - & 35 & 45 & 50 & 40 \\
\hline
\end{tabular}


growing seasons for the crops the level of observations between the different experiments is variable. Also the successive observations in one single experiment were performed over a certain period, during which water consumption could result in a decrease in water content of the vessels, thereby improving aeration (Fig. 2). The comparison of the results can thus only be based on approximate averages (Table 1 ).

Already the tentative conclusion can be made that tulip roots do not require an extra high ODR for their growth, but are within the range encountered for a variety of crops. This fact is further confirmed by some data showing the rather slow decline in root condition after increasing periods of anaerobic conditions.

Inducing these anaerobic conditions by means of $\mathrm{N}_{2}$ above the soil results in the observation that after 1 week hardly any effect on roots is visible. It is only after about 2 weeks that damage sets in, which becomes more visible after 17 days. With potatoes, the root condition already deteriorates slightly after 1 week and after 4 weeks nearly all roots have died. Flooding tulip roots results in very little damage after 1 week and occurrence of dead tips after 11 days. This result seems comparable to data obtained on peas (Cannell, 1978), where 5 days of flooding reduced dry matter production by about $1 / 6$ th and 8 days caused a $40 \%$ decrease.

The fact that the results obtained suggest that tulip roots are less sensitive than those of the potato might be partly accounted for by a difference in soil temperature during the experiments. The experiments with tulips were carried out during the winter period in an only slightly heated glasshouse with soil temperatures in the vessels in the range of $4-10^{\circ} \mathrm{C}(277-283 \mathrm{~K})$. In the potato experiments during the spring-autumn period the soil temperatures were at a higher level, about $293 \mathrm{~K}$.

General experience has shown that at higher soil temperatures the resistance to oxygen deficiency is lower on account of the higher consumption as a result of increased root metabolism, inducing faster depletion and more severe stress. But as tulips are planted in autumn and are harvested early in season (July), their roots will never be exposed to high soil temperatures.

Next, we may examine our results in relation to determining a generally valid limiting ODR value for application in the field. In single experiments - with one type of soil and variations in $\mathrm{O}_{2}$ content of the air above the soil - the roots will show a clear response with regard to growth downwards (Fig. 3). But as has already been indicated - and more explicitly elucidated in McIntyre's (1966) extensive and critical review of this method - the values obtained in these experiments are not generally valid. This was corroborated by the variation in levels obtained in field work.

Table 2. Some limiting ODR values and rooting depths attained at two different soil densities.

\begin{tabular}{|c|c|c|c|c|c|c|c|}
\hline \multicolumn{2}{|l|}{ Soil density $\left(\mathrm{g} \mathrm{cm}^{-3}\right)$} & \multicolumn{4}{|c|}{ Potato } & \multicolumn{2}{|c|}{ Pea } \\
\hline \multirow[t]{2}{*}{1.20} & root depth $(\mathrm{cm})$ & 59 & 54 & 49 & 21 & 46 & 20 \\
\hline & ODR $(\mu \mathrm{A})$ & 21 & 33 & 25 & 40 & 22 & 50 \\
\hline \multirow[t]{2}{*}{1.30} & root depth $(\mathrm{cm})$ & 49 & 45 & 21 & 16 & 42 & 17 \\
\hline & ODR $(\mu \mathrm{A})$ & 21 & 24 & 59 & 30 & 31 & 36 \\
\hline
\end{tabular}


Table 3. Relative rooting depths and corresponding mineral contents of the plants as affected by normal $(=100)$ and low $\mathrm{O}_{2}$ supply.

\begin{tabular}{lrrrrr}
\hline Plant & Rooting depth & N & P & K & Ca \\
Potato & 100 & & & & 100 \\
Potato & 45 & 100 & 100 & 103 & 100 \\
& 100 & 100 & 105 & 100 & 94 \\
Tomato & 33 & 80 & 78 & 60 & 100 \\
& 100 & 100 & 100 & 100 & 100 \\
Tulip & 63 & 96 & 64 & 42 & 107 \\
& 100 & 100 & 100 & 100 & 100 \\
& 70 & 88 & 89 & 74 & 87 \\
\hline
\end{tabular}

In these experiments higher values were always obtained in the sandy soils (Table 1). Although in some experiments two soil densities were compared, no constant requirement for increased $\mathrm{O}_{2}$ supply at higher density was noticed (Table 2).

Plant analysis yielded data which allow a few remarks on an old problem to be made with regard to the question which of the two processes is the more sensitive to a reduction in oxygen supply rate: either root growth or the function of ion absorption. Although in these experiments the results are not all exactly the same and quite some variation occurs, the general tendency is that the $\mathrm{Ca}$ content is variable and that the $\mathbf{N}$ content may often decrease somewhat in the smaller plants at low oxygen supply levels (Table 3 ). The reaction of the $\mathrm{P}$ and $\mathrm{K}$ content to the imposed variation in $\mathrm{O}_{2}$ supply is far more pronounced. In most cases a lowering of their content is correlated with the shallower rooting, lesser root weights and reduction in size of the plant. Deficiency symptoms were, however, never observed.

This difference in behaviour between these two groups of elements can be explained in the following manner. The supply of $\mathrm{P}$ and $\mathrm{K}$ to the plant is highly dependent on the root surface area involved in uptake, for it is only the active uptake process that lowers the ion concentration at the root surface, which condition is necessary to induce further supply by diffusion and desorption. The amounts of $\mathbf{P}$ and $\mathbf{K}$ brought towards the root surface along with the attracted soil solution - of which the water is used in transpiration - is far below the plant requirement in general (Barber, 1962). The restriction in root development as the result of low oxygen treatment can thus be held responsible for the lowered uptake.

The mode of supply of $\mathrm{Ca}$ and $\mathrm{NO}_{3}$ is mainly by convection (mass flow) in the soil solution attracted. As a result it is not so strictly related to root surface or to the densely rooted volume of soil. In our experiments, reasonable amounts could have been delivered by the lower soil layers along with the stream of water moving upward. The higher $\mathrm{N}$ content that sometimes occurs at the low $\mathrm{O}_{2}$ treatment can be seen as evidence of the fact that the capacity for uptake of the root systems of varying development is not impaired. The conclusion can be that the low oxygen treatments will allow root penetration downwards till the limiting ODR level is 
reached, but that within this range of possible root growth conditions uptake capacity remains unaltered. So the uptake process proper does not seem to require a higher supply rate of $\mathrm{O}_{2}$ than root growth.

This explanation is in line with other observations in our institute. Schuurman (1966) found the same type of response when restriction of root growth was effected by variations in soil density. Earlier experiments of our own (Wiersum, 1967) on the utilization of subsoil also clearly demonstrated that uptake stays at the same level of intensity, till the aeration status is so low as to impair root development.

The decrease in plant size under the lowered oxygen supply conditions may be the overall result of restricted supply of minerals like $\mathrm{P}$ and $\mathrm{K}$ and of water.

\section{References}

Barber, S. A., 1962. A diffusion and mass-flow concept of soil nutrient availability. Soil Sci. 93: 39-49.

Boekel, P., 1971. Soil structure problems in tulip culture. Acta Hortic. 23: 338-343.

Cannell, R. Q., R. K. Belford, K. Gales \& G. R. Beetlestone, 1978. Lysimeter studies on the effects of short-term waterlogging on crop growth. A. Rep. Letcombe Lab. 1977: 57-59.

Lemon, E. R. \& A. E. Erickson, 1955. Principle of the platinum micro-electrode as a method of characterizing soil aeration. Soil Sci. 79: 383-392.

McIntyre, D. S., 1966. Characterizing soil aeration with a platinum micro-electrode, I and II. Aust. J. Soil Res. 4: 95-103, 103-115.

Schuurman, J. J., 1966. Effects of soil density on root and top growth of oats. Proc. Int. Sci. Symp. (22-24 June 1966, Brno, Czechoslovakia). 103-120.

Schuurman, J. J., J. J. H. de Boer \& L. Knot, 1974. De reactie van wortelgroei, opname en spruitgroei van haver op dichtheid van zandgrond. Landbouwk. Tijdschr. 86: 256-265.

Stolzy, L. H. \& J. Letey, 1964. Measurement of O.D.R. with the platinum micro-electrode. III. Correlation of plant response to soil oxygen diffusion rates. Hilgardia 35: 567-576.

Wiersum, L. K., 1960. Some experiences in soil aeration measurements and relationships to depth of rooting. Neth. J. agric Sci. 8: 245-252.

Wiersum, L. K., 1967. Potential subsoil utilization by roots. Pl. Soil 27: 383-400.

Wiersum, L. K., 1971. Tulip root behaviour and aeration requirements. Acta Hortic. 23: 318-325. 